\title{
Study of structure and magnetic properties of Ni-Zn ferrite nano-particles synthesized via co-precipitation and reverse micro-emulsion technique
}

\author{
M. Abdullah Dar · Jyoti Shah $\cdot$ W. A. Siddiqui • \\ R. K. Kotnala
}

Received: 13 March 2013/Accepted: 23 May 2013/Published online: 22 June 2013

(c) The Author(s) 2013. This article is published with open access at Springerlink.com

\begin{abstract}
Nano-crystalline $\mathrm{Ni}-\mathrm{Zn}$ ferrites were synthesized by chemical co-precipitation and reverse microemulsion technique with an average crystallite size of 11 and $6 \mathrm{~nm}$, respectively. The reverse micro-emulsion method has been found to be more appropriate for nanoferrite synthesis as the produced particles are monodisperse and highly crystalline. Zero-field cooled and field cooled magnetization study under different magnetic fields and magnetic hysteresis loops at different temperatures have been performed. The non-saturated M-H loops, absence of hysteresis, and coercivity at room temperature are indicative of the presence of super paramagnetic and singledomain nano-particles for both the materials. In sample ' $a$ ', the blocking temperature $\left(T_{\mathrm{B}}\right)$ has been observed to decrease from 255 to $120 \mathrm{~K}$ on increasing the magnetic field from 50 to $1,000 \mathrm{Oe}$, which can be attributed to the reduction of magneto crystalline anisotropy constant. The $M_{\mathrm{S}}$ and coercivity were found to be higher for sample ' $\mathrm{a}$ ' as compared with sample 'b' since surface effects are neglected on increasing the crystallite size.
\end{abstract}

Keywords Micro-emulsion · Nano-particles · Super-paramagnetism $\cdot$ Blocking temperature . Coercivity

M. Abdullah Dar · W. A. Siddiqui

Department of Applied Sciences and Humanities,

Jamia Millia Islamia University,

New Delhi 110025, India

M. Abdullah Dar · J. Shah · R. K. Kotnala (凹)

National Physical Laboratory, Dr. K. S. Krishnan Road,

New Delhi 110012, India

e-mail: rkkotnala@mail.nplindia.org

\section{Introduction}

Nano-crystalline ferrites are materials of considerable interest because of their unique dielectric, magnetic, and optical properties, which make them appealing both from the scientific and technological points of view (Wang et al. 1998; Abdullah Dar et al. 2010). These magnetic materials are the basis of a very active research field due to the new phenomena taking place at the nano-scale level as a consequence of the interplay of quantum, finite-size, surface, and interfacial effects. Spinel ferrite nano-particles with a high surface area have many technical applications in several fields such as high-density information storage, ferro-fluids, catalysts, drug targeting, hyperthermia, magnetic separation, and magnetic resonance imaging (Cannas et al. 2010). The key questions in these systems are how these nano-structures modify their magnetic and electronic properties and how one can take advantage of those new properties to improve the applications. Consequently, understanding and controlling the effects of the nanostructures on the properties of the particles have become increasingly relevant issues for technological applications (Batlle et al. 2011).

The study of magnetic behavior of ferrites has attracted considerable attention from past few decades, in particular because deviations from bulk behavior have been widely reported for particle sizes below about $100 \mathrm{~nm}$ range. This is because of finite-size effects and the increasing fraction of atoms lying at the surface with lower atomic coordination than in the core as the size of the particle decreases, thus giving rise to a significant decrease in the particle magnetization (Batlle et al. 2011). The surface spin disorder due to symmetry breaking at the surface leads to a smaller saturation magnetization, high field differential susceptibility, extremely high closure fields, and shifted 
hysteresis loops after field cooling the sample, together with glassy behavior. This has been explained in terms of the existence of a surface layer of disordered spins that freeze in a spin-glass-like state due to magnetic frustration, yielding both an exchange field acting on the ordered core and an increase in the particle anisotropy. The most obvious and heavily studied finite-size effect is super-paramagnetism. The basic principle is that the magnetic anisotropy energy which keeps a particle magnetized in a particular direction is generally proportional to the particle volume. Therefore, below a critical size at room temperature, the thermal fluctuations are sufficient to rotate the particle magnetization, hence demagnetizing an assembly of such particles. Although this is a well-studied effect, it is understood only on a phenomenological level.

Recently, considerable attention has been paid on ferrites of different morphology and their shape- and sizedependent properties as well their corresponding applications were investigated. Both the physical and chemical methods have been developed for the synthesis of ferrite nano-structures of various morphologies. The chemical methods have advantages over the physical one such as low cost, reaction taking place at low temperature, and largescale production possibility. It is widely appreciated that the cation distribution in spinel ferrites upon which many physical and chemical properties depend is a complex function of processing parameters and depends on the preparation method of the material (Sepelak et al. 2007). Selection of an appropriate method is the key factor to obtain ferrites of high quality. Various processing methods have been developed to obtain nano-crystalline ferrites such as hydrothermal synthesis, chemical co-precipitation, polymeric precursor techniques, sol-gel, shock wave, spray drying, sono-chemical process and mechanical alloying (Sivakumar et al. 2011; Kotnala et al. 2010). It is well known that different routes produce different microstructures and crystal sizes. Therefore, based on the ease and reproducibility, chemical co-precipitation method is widely used but it leads to the precipitation of nano-crystals with a relatively broad size distribution (Zhang et al. 1998). In the present study a reverse micro-emulsion technique has been selected for the synthesis of nano-crystals. Reverse micelles, which are essentially nano-sized aqueous droplets that exist in micro-emulsions with certain compositions, are known to present an excellent medium for the synthesis of nano-particles. The particles produced by this method are generally very fine, mono-disperse, morphologically controlled, and highly crystalline as compared with the other processes ( $\mathrm{Li}$ and Park 1999; Yener and Giesche 2001). Current interest to synthesize nano-structured nickel-zinc ferrite particles is due to its low value of coercivity, high resistivity, and high saturation magnetization similar to that of magnetite (Thakur et al. 2007). In this work, we have reported the synthesis of nano-crystalline sized mixed ferrite system, namely $\mathrm{Ni}_{0.7} \mathrm{Zn}_{0.3} \mathrm{Fe}_{2} \mathrm{O}_{4}$, by chemical co-precipitation and reverse micro-emulsion method. The structure and morphology analysis has been carried out using X-ray diffraction (XRD) and transmission electron microscopy (TEM). The magnetic properties of the synthesized samples were measured using vibrating sample magnetometer at different temperatures and discussed in detail.

\section{Experimental}

Synthesis of $\mathrm{Ni}_{0.7} \mathrm{Zn}_{0.3} \mathrm{Fe}_{2} \mathrm{O}_{4}$ ferrite nano-crystals by reverse micro-emulsion method

The reverse micro-emulsion system consists of cyclohexane as an oil phase, Cetyl-tri-methyl-ammonium bromide (CTAB) as a surfactant, and iso-amyl-alcohol as the co-surfactant phase. All the chemicals in this work were of analytical grade and used as received without further purification. Reverse micro-emulsions were prepared by adding to $10.20 \mathrm{~g}$ of CTAB, $12.81 \mathrm{ml}$ of iso-amyl-alcohol, and $30.48 \mathrm{ml}$ of cyclo-hexane with $5.5 \mathrm{wt} \%$ of an aqueous solution of the reactants, corresponding to the desired value of water/[CTAB] ratio being equal to 10.12 . The reverse micro-emulsions were sonicated until clear solution was formed. Two reverse micro-emulsions were prepared for the synthesis of $\mathrm{Ni}_{0.7} \mathrm{Zn}_{0.3} \mathrm{Fe}_{2} \mathrm{O}_{4}$ ferrite nano-crystals, one containing the metal salts prepared by mixing stoichiometric amounts of $0.2 \mathrm{M} \mathrm{Fe}\left(\mathrm{NO}_{3}\right)_{3} \cdot 9 \mathrm{H}_{2} \mathrm{O}, \quad 0.07 \mathrm{M}$ $\mathrm{Ni}\left(\mathrm{NO}_{3}\right)_{2} \cdot 6 \mathrm{H}_{2} \mathrm{O}$ and $0.03 \mathrm{M} \mathrm{Zn}\left(\mathrm{NO}_{3}\right)_{2} \cdot 6 \mathrm{H}_{2} \mathrm{O}$. In the second reverse micro-emulsion, $0.1 \mathrm{M}$ aqueous $\mathrm{NaOH}$ solution was added as water phase under similar conditions. The two solutions were mixed together quickly with vigorous stirring at constant temperature $\left(80^{\circ} \mathrm{C}\right)$ and $\mathrm{pH}$ of the resulting solution was maintained at 9 . The resulting solution was continuously stirred for another $2 \mathrm{~h}$ for reaction completion. An equal volume of acetone and isopropanol was added to the resulting solution and centrifuged to separate the solid product. The product obtained was washed several times with water and acetone to ensure the complete removal of surfactant molecules from the product. Finally, the product obtained was dried in an air oven at $100{ }^{\circ} \mathrm{C}$ for $12 \mathrm{~h}$.

Synthesis of $\mathrm{Ni}_{0.7} \mathrm{Zn}_{0.3} \mathrm{Fe}_{2} \mathrm{O}_{4}$ ferrite nano-crystals by chemical co-precipitation method

In a typical procedure, nano-crystals of $\mathrm{Ni}_{0.7} \mathrm{Zn}_{0.3} \mathrm{Fe}_{2} \mathrm{O}_{4}$ ferrite were synthesized by chemical co-precipitation of $\mathrm{Fe}\left(\mathrm{NO}_{3}\right)_{3} \cdot 9 \mathrm{H}_{2} \mathrm{O}, \mathrm{Ni}\left(\mathrm{NO}_{3}\right)_{2} \cdot 6 \mathrm{H}_{2} \mathrm{O}$ and $\mathrm{Zn}\left(\mathrm{NO}_{3}\right)_{2} \cdot 6 \mathrm{H}_{2} \mathrm{O}$ in an alkaline medium at constant $\mathrm{pH}$ of 9 . The stock 
solutions of all the precursors were prepared with same concentration as used in the reverse micro-emulsion method. The stoichiometric amounts of $\mathrm{Fe}\left(\mathrm{NO}_{3}\right)_{3} \cdot 9 \mathrm{H}_{2} \mathrm{O}$, $\mathrm{Ni}\left(\mathrm{NO}_{3}\right)_{2} \cdot 6 \mathrm{H}_{2} \mathrm{O}$, and $\mathrm{Zn}\left(\mathrm{NO}_{3}\right)_{2} \cdot 6 \mathrm{H}_{2} \mathrm{O}$ were mixed together. Then this mixture was poured into $0.1 \mathrm{M} \mathrm{NaOH}$ solution under constant stirring and at a constant temperature of $80{ }^{\circ} \mathrm{C}$. The resulting mixture was continuously stirred for $2 \mathrm{~h}$ at same temperature and $\mathrm{pH}$ of 9 . The resulting precipitate were then filtered off and washed several times with methanol and double distilled water followed by drying in an air oven at $100{ }^{\circ} \mathrm{C}$ for $12 \mathrm{~h}$.

\section{Characterization}

X-ray diffraction (XRD) analysis of both the samples were carried out using Rigaku Miniflex (Step size $=0.02$ ) with $\mathrm{CuK} \alpha$ radiation of wavelength $\lambda=1.5406 \AA$. The particle size, morphology, and SAED patterns of both the ferrite samples were determined by high-resolution transmission electron microscopy (HRTEM) using JEM 200CX model. The variation of magnetization as a function of field, up to a maximum field of $5 \mathrm{k}$ Oe at different temperatures (80-300 K) was measured using vibrating sample magnetometer (Lakeshore, 7304). The zero-field-cooled (ZFC) measurement was carried out by cooling the sample in a zero magnetic field from 300 to $80 \mathrm{~K}$. The magnetization was measured while increasing the temperature from 80 to $300 \mathrm{~K}$ in a magnetic field of 50 Oe. Field cooled (FC) measurements were performed in the same manner with the difference being that the cooling of the samples was done in the same field of 50 Oe. In order to study the effect of applied magnetic field on the blocking temperature $\left(T_{\mathrm{B}}\right)$ and hence on the magneto-crystalline anisotropy, ZFC-FC measurements were performed at two different magnetic fields (50 and 1,000 Oe).

\section{Results and discussion}

X-ray diffraction pattern of $\mathrm{Ni}_{0.7} \mathrm{Zn}_{0.3} \mathrm{Fe}_{2} \mathrm{O}_{4}$ ferrite nanocrystals synthesized via chemical co-precipitation and reverse micro-emulsion technique are presented in Fig. 1a, $\mathrm{b}$, respectively. The sharp peaks from diffraction pattern show the crystalline nature of these samples. All the diffraction peaks could be ascribed to the reflections of (220), (311), (400), (422), (511), and (440) planes which could be indexed to a face-centered cubic $\mathrm{Ni}-\mathrm{Zn}$ ferrite phase. The reflections were comparatively broader, revealing that the as-prepared crystals are small in size. According to the Scherrer's equation (Verma et al. 2010), the average crystallite size determined from the half-width of the most intense peak (311) was found to be 10.3 and $5.7 \mathrm{~nm}$ for sample ' $a$ ' and ' $b$ ', respectively. The difference in the

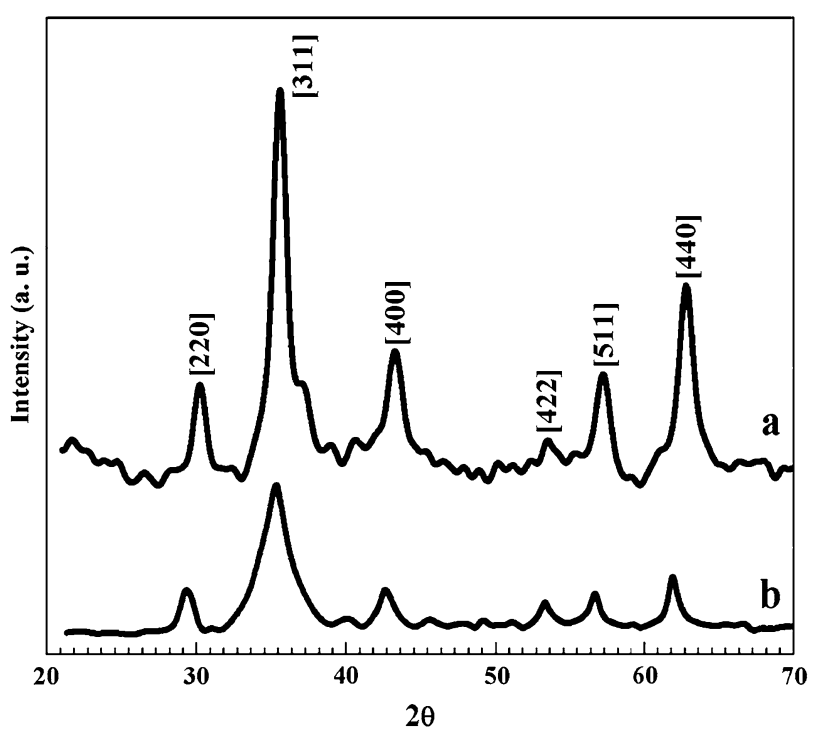

Fig. 1 XRD pattern of $\mathrm{Ni}_{0.7} \mathrm{Zn}_{0.3} \mathrm{Fe}_{2} \mathrm{O}_{4}$ ferrite nano-crystals obtained from a co-precipitation and $\mathbf{b}$ reverse micro-emulsion method

crystallite size was probably due to the difference in preparation conditions followed in this work. It gave rise to different rates of nucleation, growth, coarsening and agglomeration processes, and hence favoring the variation in crystallite size. The X-ray diffraction pattern of $\mathrm{Ni}_{0.7} \mathrm{Zn}_{0.3} \mathrm{Fe}_{2} \mathrm{O}_{4}$ ferrite nano-crystals obtained from chemical co-precipitation method exhibits the more intense peaks, indicating their higher crystallinity. The obtained lattice constant values for the nano-crystals of sample ' $a$ ' and ' $b$ ' prepared by chemical precipitation and reverse micro-emulsion method were found to be 8.35 and $8.41 \AA$, respectively.

Figure $2 \mathrm{a}, \mathrm{b}$ shows the transmission electron microscopy (TEM) images of $\mathrm{Ni}_{0.7} \mathrm{Zn}_{0.3} \mathrm{Fe}_{2} \mathrm{O}_{4}$ ferrite nanocrystals synthesized using chemical co-precipitation and reverse micro-emulsion technique, respectively. The particle size distribution and their selected area electron diffraction (SAED) patterns are included as insets in Fig. 2a, b. The SAED pattern correspond to various diffractions of both samples. It confirmed spinel phase formation consistent with XRD pattern. It is evident from Fig. 2a that the particles obtained by chemical co-precipitation method are irregular in shape and the average particle size $(\sim 11 \mathrm{~nm})$ obtained from TEM is bigger than that observed from X-ray line broadening technique. This may be due to the agglomeration of fine particles. Reverse micro-emulsion process has resulted in a narrower, monodisperse and nearly spherical particle size distribution with reduced average diameter $(\sim 6 \mathrm{~nm})$ as shown in Fig. 2b. In reverse micro-emulsion synthesized samples, the average crystallite size obtained from Scherer's equation were found to be in good agreement with that 


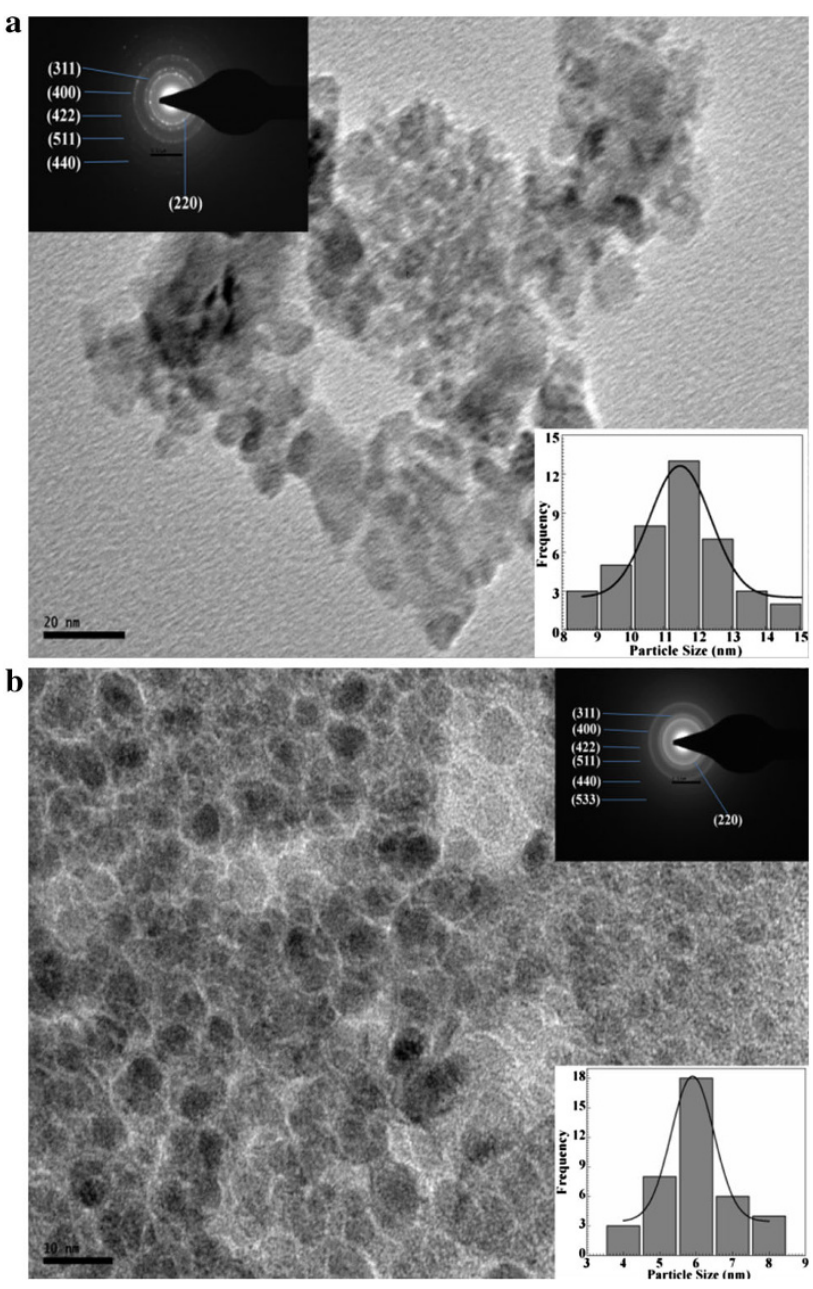

Fig. 2 TEM images of nano-crystals obtained from a co-precipitation and $\mathbf{b}$ reverse micro-emulsion method. The inset in $\mathbf{a}$ and $\mathbf{b}$ shows SAED pattern and histogram of the particle size distribution

obtained from TEM, which means that each particle behaves as a single crystal.

The analysis of XRD and TEM results divulges that the nano-crystals obtained from the abovementioned methods have different crystallite size. This difference in crystallite size has been attributed to the different preparation conditions followed here, which gave rise to different rate of ferrite formation and hence favoring the variation in crystallite size. The reverse micro-emulsion technique allows the control over both shape and size via the structure of the surfactant assemblies. This is a technique, which allows the preparation of ultrafine nano-particles within the particle diameter of 1-100 nm (Vaidyanathan et al. 2007). The reverse micro-emulsion solutions are mostly transparent, isotropic liquid media with nano-sized water droplets that are dispersed in the continuous oil phase and stabilized by surfactant molecules at the water/oil interface. The nanoenvironment created by surfactant-covered water pools not only acts as nano-reactors for processing reactions but also inhibits the process of aggregation. It could be due to the surfactant molecules that could adsorb on the particle surface, when the particle size approaches that of the water pool. As a result, the particles obtained by this technique are generally very fine, mono-disperse, morphologically controlled, and highly crystalline (Li and Park 1999; Yener and Giesche 2001).

Figure $3 \mathrm{a}, \mathrm{b}$ represents the $\mathrm{M}-\mathrm{H}$ curves for sample ' $\mathrm{a}$ ' and ' $b$ ' measured in the temperature range of 80 and $310 \mathrm{~K}$ prepared by chemical co-precipitation and reverse microemulsion method, respectively. It is well known that magnetic nano-particles $<20 \mathrm{~nm}$ are usually super-paramagnetic at room temperature. Indeed, all of our measurements at room temperature are in agreement with this view, without any evidence of a significant slow down in the relaxation of the magnetic domains. Upon cooling, this super-paramagnetic behavior of the magnetization turns into a typical field dependence of the magnetic single domain, once the temperature drops below the blocking temperature $\left(T_{\mathrm{B}}\right)$. The typical characteristics of superparamagnetic behavior like absence of hysteresis and the non-attainment of saturation even up to an applied magnetic field of $5 \mathrm{k}$ Oe were observed, which is indicative of the presence of super-paramagnetic and single-domain particles for both the ferrite samples. At low temperature $(80 \mathrm{~K})$, the nano-particles of these ferrites start to indicate remanence and coercivity, and exhibit hysteretic features as shown in Fig. 3a, b. At this temperature the particles do not have adequate thermal energy to attain complete equilibrium with the applied field during the measurement time and hence hysteresis appears. The lower $M_{\mathrm{S}}$ (31 and $6.1 \mathrm{emu} / \mathrm{g}$ for ' $a$ ' and ' $\mathrm{b}$ ' sample) of these nano-crystalline ferrites compared with the multidomain bulk values of $70 \mathrm{emu} / \mathrm{g}$ (Chikazumi 1964) is attributed to the increased cation disorder and surface effects in nano-crystalline ferrites. This can be explained in terms of the core-shell morphology of nanoparticles consisting of ferri-magnetically aligned core spins and a spin-canted like surface layer. The spin disorder from the surface of the nano-particles may essentially modulate the magnetic properties of these materials, especially when the surface/volume ratio is large enough (Lee et al. 2005). When a magnetic field is applied the core magnetic moments get aligned with the applied magnetic field and at some stage the core magnetization response is exhausted while the core magnetization of the system is saturated like Langevin way. Beyond this stage, any increase in the magnetic field on the particles has an effect only on the surface layer of the particles and thus an increase in the magnetization of particles slows down. This specific state of the surface results in an absence of magnetic saturation (Thakur et al. 2009). Further, an increase in the magneto-crystalline anisotropy can result from the inter-particle interactions, which are more intense 

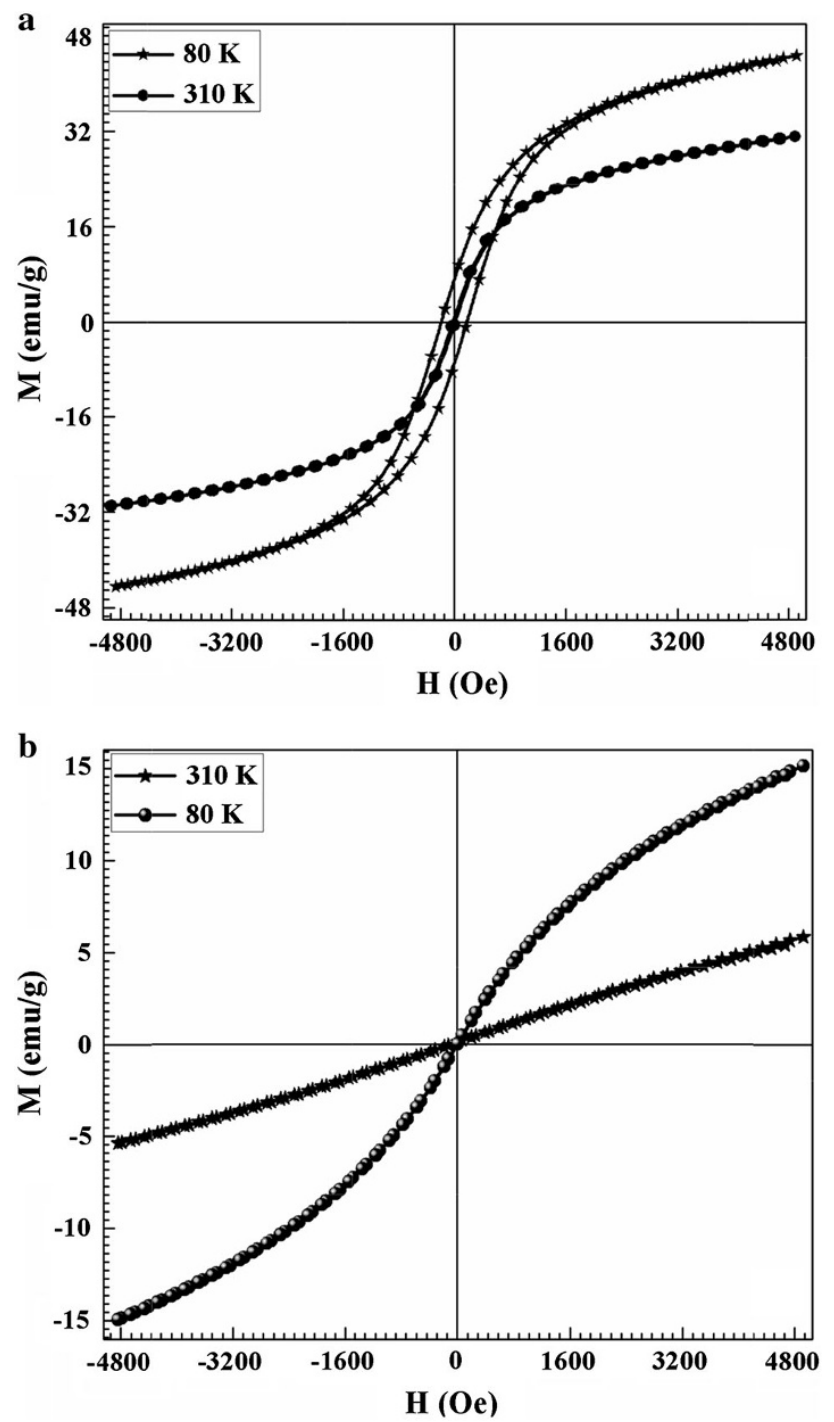

Fig. $3 \mathrm{M}-\mathrm{H}$ curves of $\mathrm{Ni}_{0.7} \mathrm{Zn}_{0.3} \mathrm{Fe}_{2} \mathrm{O}_{4}$ ferrite nano-crystals obtained from a co-precipitation and $\mathbf{b}$ reverse micro-emulsion method measured with an applied field of $5 \mathrm{k}$ Oe at 80 and $310 \mathrm{~K}$

in case of ferrites because of the super-exchange interactions. The presence of any defect on the surface leads to the weakening of these interactions inducing a large surface spin disorder (Nathani and Misra 2004). The small value of $M_{\mathrm{S}}$ can also be explained on the basis of cation redistribution in these ferrite nano-crystals. In Ni- $\mathrm{Zn}$ ferrite, $\mathrm{Zn}^{2+}$ ions occupies the tetrahedral (A) site and $\mathrm{Ni}^{2+}$ ions occupy the octahedral (B) site of the spinel lattice. But in nanoscale ferrites cations occupy lattice sites by a certain degree against their preferences in bulk materials and this inversion is dependent on particle size. As the crystallite size decreases there is a change in the degree of inversion parameters, i.e., there are more $\mathrm{Ni}^{2+}$ ions occupying A-sites and more $\mathrm{Zn}^{2+}$ ions occupying B-sites results in lowering the magnetization values for these nano-crystals (Rao et al. 2006).
Table 1 Variation of lattice parameter and coercivity (at different temperatures) of $\mathrm{Ni}_{0.7} \mathrm{Zn}_{0.3} \mathrm{Fe}_{2} \mathrm{O}_{4}$ ferrite nano-crystals

\begin{tabular}{lllllllll}
\hline Composition & Lattice parameter & \multicolumn{6}{l}{ Coercivity (Oe) } \\
\cline { 3 - 8 } $\mathrm{Ni}_{0.7} \mathrm{Zn}_{0.3} \mathrm{Fe}_{2} \mathrm{O}_{4}$ & $a(\AA)$ & $80 \mathrm{~K}$ & $100 \mathrm{~K}$ & $150 \mathrm{~K}$ & $200 \mathrm{~K}$ & $250 \mathrm{~K}$ & $300 \mathrm{~K}$ \\
\hline $\begin{array}{c}\text { Co- } \\
\text { precipitation }\end{array}$ & 8.34 & 184 & 158 & 97 & 58 & 37 & 17 \\
\begin{tabular}{c} 
Micro-emulsion \\
\hline
\end{tabular} & 8.36 & 6.6 & 6.67 & 6.8 & 7.2 & 7.6 & 7.9 \\
\hline
\end{tabular}

A low value of coercivity, observed for both the samples is shown in Table 1 . These low values of coercivity have been attributed to the particle-particle interactions among the nano-crystals owing to their extremely small size. The proximity of particles has a large effect on the hysteresis as they either become increasingly exchange coupled or show magnetostatic interactions with decreasing distance between the particles (Verdes et al. 2002). Thus the magnetization shows an increase on lowering the temperature, but the coercivity in sample ' $b$ ' follows an opposite trend, that is, it decreases with the decrease in measuring temperature. This decrease in coercivity is expected due to the lower anisotropy indicating the highly super-paramagnetic nature of the nano-crystals obtained from reverse microemulsion method (Roca et al. 2007). The Ni-Zn ferrite nano-crystals obtained from chemical co-precipitation have crystallite size $\sim 11 \mathrm{~nm}$, which is much bigger than that of reverse micro-emulsion synthesized particles. The low temperature coercive field of this small sample is very much larger for coherent magnetization reversal of randomly oriented particles, thus suggesting that the process is controlled by a different mechanism, whose strength is very much larger than that of magneto-crystalline anisotropy energy (Bates and Wohlfarth 1980). This may be attributed to the appearance of exchange anisotropy field, which clearly indicates the existence of a magnetically disordered surface layer that becomes frozen at low temperature (Caizer and Stefanescu 2002). Therefore, the observed increase in coercivity can be attributed to the extra energy required for the switching of the core spins that are pinned by the exchange interactions with the frozen spin-glass-like surface layer. The relevance of the magnetically disordered surface layer on the magnetic properties becomes less as the particle size increases and eventually disappears for large enough particles. Consequently, the value of $M_{\mathrm{S}}$ and coercivity is higher for sample ' $a$ ' as compared with sample 'b', since surface effects are neglected.

Figure $4 \mathrm{a}, \mathrm{b}$ represents the $\mathrm{ZFC}-\mathrm{FC}$ curves recorded at an applied field of 50 Oe for $\mathrm{Ni}_{0.7} \mathrm{Zn}_{0.3} \mathrm{Fe}_{2} \mathrm{O}_{4}$ nano-crystals synthesized via chemical co-precipitation and reverse micro-emulsion method, respectively. The field cooled (FC) magnetization is obtained by cooling the sample from room temperature to $80 \mathrm{~K}$ within a field of $50 \mathrm{Oe}$ and 

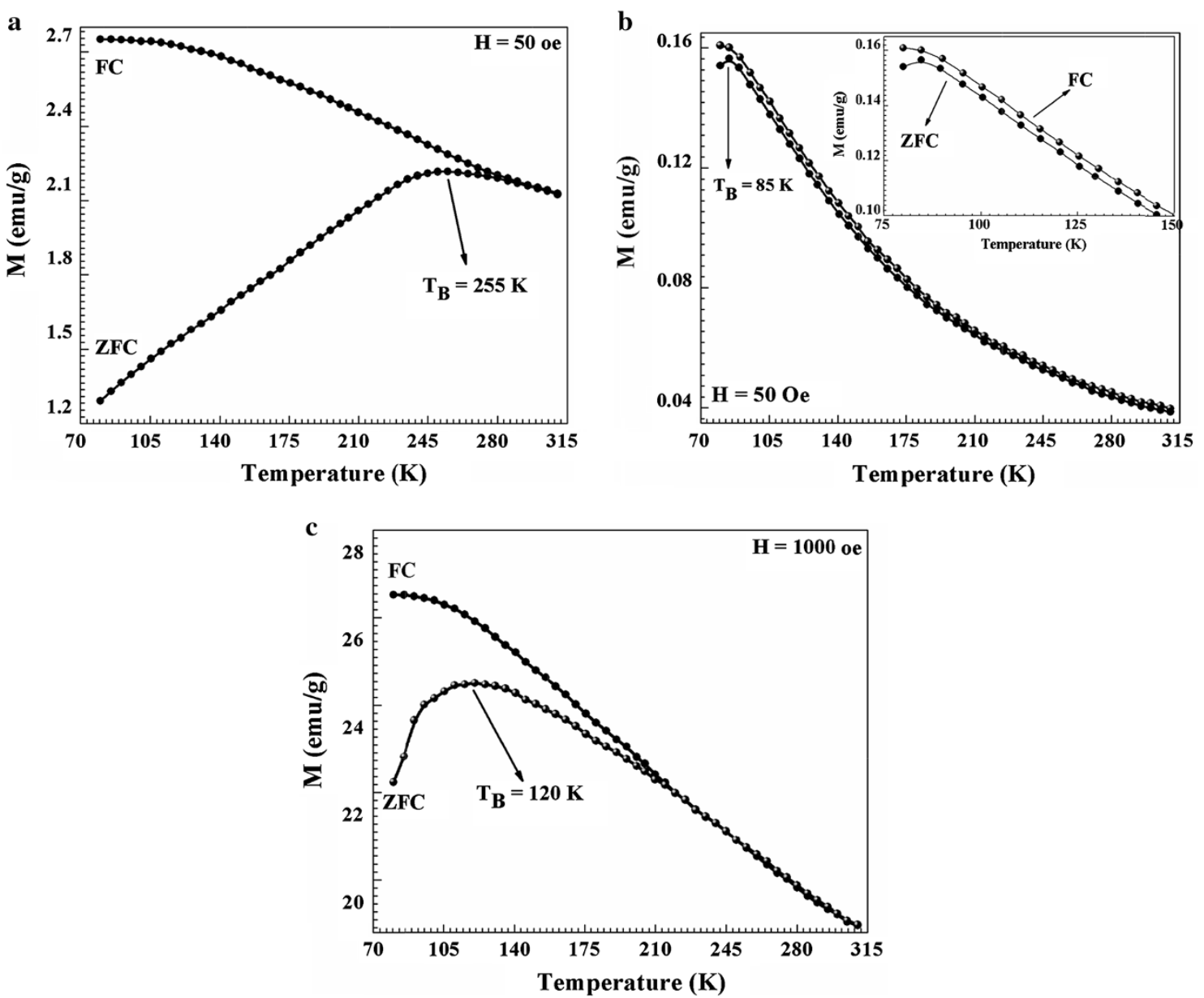

Fig. $4 \mathrm{ZFC}-\mathrm{FC}$ curves of $\mathrm{Ni}_{0.7} \mathrm{Zn}_{0.3} \mathrm{Fe}_{2} \mathrm{O}_{4}$ ferrite nano-crystals obtained from a co-precipitation, $\mathbf{b}$ reverse micro-emulsion method recorded at $50 \mathrm{Oe}$ and $\mathbf{c}$ recorded at $1,000 \mathrm{Oe}$ for sample a

simultaneously measuring the magnetization while increasing the temperature. The zero field cooled (ZFC) magnetization is obtained by cooling the sample in zerofield, then warming within the field of $50 \mathrm{Oe}$, and simultaneously measuring the magnetization while increasing the temperature. From Fig. $4 a, b$ it is clear that on increasing temperature the ZFC magnetization increases, reaches maximum at the blocking temperature $\left(T_{\mathrm{B}}\right)$, and then again decreases. The $T_{\mathrm{B}}$ is defined as the temperature at which the nano-particle moments do not relax (are blocked) during the time scale of the measurement. The spins are able to flip only above $T_{\mathrm{B}}$ and the ZFC magnetization then coincides with the FC magnetization (Neel 1953). At temperatures above $T_{\mathrm{B}}$, the thermal energy is larger than the magnetic energy barrier and thus the materials shows super-paramagnetism following the CurieWeiss law. The blocking temperature of Ni-Zn ferrite nano-crystals were found to be 225 and $85 \mathrm{~K}$ for sample ' $\mathrm{a}$ ' and 'b', respectively, as is evident from Fig. 4a, b. In order to study the variation of magnetic field on the blocking temperature, ZFC-FC measurements were also performed with an applied filed of 1,000 Oe for sample ' $a$ ' as shown in Fig. 4c. From the curves it is clear that the blocking temperature shifts to lower temperature (225-120 K) with the increase of applied field. It could be attributed to the reduction of magneto-crystalline anisotropy constant. In FC measurements, the magnetization direction of nanoparticles is frozen to the direction of applied field when nano-particles are cooled from room temperature to $80 \mathrm{~K}$. At $80 \mathrm{~K}$, the anisotropy energy barrier prevents the magnetic moments from flipping and hence a maximum magnetization has been recorded at this temperature. The divergence between the FC and ZFC curve is a characteristic feature of super-paramagnetism and results from the anisotropy energy barrier of the nano-particles. In the ZFC process the nano-particles need to overcome the anisotropy energy barrier, as the moments of the nano-particles are oriented along their easy axis. The anisotropy energy barrier does not have an effect on the FC curve, as the nanoparticle moments are aligned with the field during the cooling process. The FC curve appears to be nearly flat below the blocking temperature, as compared with the 
increasing behavior characteristic of super-paramagnetic systems, which indicates the existence of strong interactions among the nano-particles. However, this feature has been found not to be exclusive of spin glass, but also shared by nano-particle systems with random anisotropy and strong dipole-dipole interactions (Jiao et al. 2002). When a magnetic field is applied to a magnetic material, the domain walls rotate in such a way that its multidomain structure changes towards a single-domain structure with increase in the field. In addition to the extrinsic factors such as defects and lattice strains, another very important factor that plays a crucial role in determining the domain wall motion is the magneto-crystalline anisotropy energy.

The magneto-crystalline anisotropy energy of a singledomain particle is given by $E_{\mathrm{A}}=K V \sin ^{2} \theta$, where $K$ is the magneto-crystalline anisotropy constant, $V$ is the volume of the nano-particle, and $\theta$ is the angle between the direction of magnetization and the easy axis of the nano-particle. $\mathrm{KV}$ is the anisotropy energy barrier for the reversal of magnetic moment (Goya 2004). The cation distribution in a particular ferrite also plays a dominant role in the resultant blocking temperature.

The reversal or switching time, called the Neel relaxation time, is given by the following relation (1):

$\tau=\tau_{o} \exp \left(E_{\mathrm{A}} / k_{\mathrm{B}} T_{\mathrm{B}}\right)$

or

$E_{\mathrm{A}}=\ln \left(\tau / \tau_{o}\right) k_{\mathrm{B}} T_{\mathrm{B}}$,

where $\tau$ is the super-paramagnetic relaxation time (60 s), $\tau_{\mathrm{o}}$ is a relaxation time constant $\left(\sim 10^{-10} \mathrm{~s}\right)$, and $T_{\mathrm{B}}$ is the blocking temperature. The magneto-crystalline anisotropy constant is larger for nano-crystalline materials than the bulk one and it increases with the decrease in particle size as well as decreases with the increase of applied magnetic field. Assuming the particles to be spherical, the magnetocrystalline anisotropy constant for sample ' $a$ ' was found to decrease from $12.63 \times 10^{5}$ to $5.94 \times 10^{5} \mathrm{erg} / \mathrm{cm}^{3}$ on increasing the magnetic field from 50 to $1,000 \mathrm{Oe}$, respectively. The magneto-crystalline anisotropy constant was found to be $25.94 \times 10^{5} \mathrm{erg} / \mathrm{cm}^{3}$ for sample ' $\mathrm{b}$ ' at an applied field of $50 \mathrm{Oe}$, which is extremely higher than that of bulk ferrites.

\section{Conclusions}

A novel and facile route of reverse micro-emulsion method has been presented here for the synthesis of $\mathrm{Ni}-\mathrm{Zn}$ ferrite nano-crystals with narrow particle size distribution. In this method we have obtained fine, mono-disperse, and nearly spherical nano-particles in comparison with the irregularly shaped particles obtained by the co-precipitation method.
The presence of surfactant molecules in the reverse micelles which to some extent act as capping agents may serve to prevent agglomeration of the particles. The saturation magnetization of $\mathrm{Ni}-\mathrm{Zn}$ ferrite nano-crystals prepared by reverse micro-emulsion technique $(6.1 \mathrm{emu} / \mathrm{g})$ is less than those prepared by co-precipitation (31 emu/g) and their bulk counter parts $(70 \mathrm{emu} / \mathrm{g})$. This can be explained in terms of the core-shell morphology of the nano-particles consisting of ferrimagnetically aligned core spins and a spin-canted like surface layer. In sample 'a', the coercivity has been observed to decrease with temperature; however, a reverse trend was observed in the reverse micro-emulsion synthesized sample. The anisotropy has been observed to increase substantially with the decrease in particle size as well as decreases with the increase of applied magnetic field.

Acknowledgments One of the authors (M. Abdullah Dar) is highly grateful to CSIR for their financial assistance through grant No. 124069/2K11/1.

Open Access This article is distributed under the terms of the Creative Commons Attribution License which permits any use, distribution, and reproduction in any medium, provided the original author(s) and the source are credited.

\section{References}

Abdullah Dar M, Batoo KM, Verma V, Siddiqui WA, Kotnala RK (2010) Synthesis and characterization of nano-sized pure and Aldoped lithium ferrite having high value of dielectric constant. J Alloy Compd 493:553

Bates G (1980) In: Wohlfarth EP (ed) Ferromagnetic materials, vol 2. North-Holland, Amsterdam, p 442

Batlle X, Perez N, Guardia P, Iglesias O, Labarta A, Bartolome F, Garcia LM, Bartolome J, Roca AG, Morales MP, Serna CJ (2011) Recent advances in magnetic nanoparticles with bulk-like properties, J Appl Phys 109: 07B524

Caizer C, Stefanescu M (2002) Magnetic characterization of nanocrystalline $\mathrm{Ni}-\mathrm{Zn}$ ferrite powder prepared by the glyoxylate precursor method. J Phys D 35:3035

Cannas C, Ardu A, Peddis D, Sangregorio C, Piccaluga G, Musinu A (2010) Surfactant-assisted route to fabricate $\mathrm{CoFe}_{2} \mathrm{O}_{4}$ individual nanoparticles and spherical assemblies. J Coll Interface Sci 343:415

Chikazumi S (1964) Physics of Magnetism. Wiley, New York, p 498

Goya GF (2004) Magnetic interactions in ball-milled spinel ferrites. J Mater Sci 39:5045

Jiao X, Chen D, Hu Y (2002) Hydrothermal synthesis of nanocrystalline $\mathrm{M}_{x} \mathrm{Zn}_{1-x} \mathrm{Fe}_{2} \mathrm{O}_{4}(\mathrm{M}=\mathrm{Ni}, \mathrm{Mn}, \mathrm{Co} ; x=0.40-0.60)$ powders. Mater Res Bull 37:1583

Kotnala RK, Abdullah Dar M, Verma V, Singh AP, Siddiqui WA (2010) Minimizing of power loss in $\mathrm{Li}-\mathrm{Cd}$ ferrite by nickel substitution for power applications. J Magn Magn Mater 322:3714

Lee Y, Lee J, Bae CJ, Park JG, Noh HJ, Park JH, Hyeon T (2005) Large-scale synthesis of uniform and crystalline magnetite nanoparticles using reverse micelles as nanoreactors under reflux conditions. Adv Funct Mater 15:503

Li Y, Park CW (1999) Particle size distribution in the synthesis of nanoparticles using microemulsions. Langmuir 15:952 
Nathani H, Misra RDK (2004) Surface effects on the magnetic behavior of nanocrystalline nickel ferrites and nickel ferritepolymer nanocomposites. Mater Sci Eng B 113:228

Neel L (1953) Thermoremanent magnetization of fine powders. Rev Mod Phys 25:293

Rao BP, Kumar AM, Rao KH, Murthy YLN, Caltun OF, Dumitru I, Spinu L (2006) Synthesis and magnetic studies of Ni-Zn ferrite nanoparticles. J Optoelectr Adv Mater 8:1703

Roca AG, Marco JF, del Puerto Morales M, Serna CJ (2007) Effect of nature and particle size on properties of uniform magnetite and maghemite nanoparticles. J Phys Chem C 111:18577

Sepelak V, Bergmann I, Feldhoff A, Heitjans P, Krumeich F, Menzel D, Litterst FJ, Campbell SJ, Becker KD (2007) Nanocrystalline nickel ferrite, $\mathrm{NiFe}_{2} \mathrm{O}_{4}$ : mechanosynthesis, nonequilibrium cation distribution, canted spin arrangement, and magnetic behavior. J Phys Chem C 111:5026

Sivakumar P, Ramesh R, Ramanand A, Ponnusamy S, Muthamizhchelvan C (2011) Synthesis and characterization of $\mathrm{NiFe}_{2} \mathrm{O}_{4}$ nanosheet via polymer assisted co-precipitation method. Mater Lett 65:483

Thakur S, Katyal SC, Singh M (2007) Structural and magnetic properties of nano nickel-zinc ferrite synthesized by reverse micelle technique. Appl Phys Lett 91: 262501
Thakur S, Katyal SC, Singh M (2009) Structural and magnetic properties of nano nickel-zinc ferrite synthesized by reverse micelle technique. J Magn Magn Mater 321:1

Vaidyanathan G, Sendhilnathan S, Arulmurugan R (2007) Structural and magnetic properties of $\mathrm{Co}_{1-x} \mathrm{Zn}_{x} \mathrm{Fe}_{2} \mathrm{O}_{4}$ nanoparticles by coprecipitation method. J Magn Magn Mater 313:293

Verdes C, Thompson SM, Chantrell RW, Stancu AL (2002) Computational model of the magnetic and transport properties of interacting fine particles. Phys Rev B 65:174417

Verma V, Abdullah Dar M, Pandey V, Singh A, Annapoorni S, Kotnala RK (2010) Magnetic properties of nano-crystalline $\mathrm{Li}_{0.35} \mathrm{Cd}_{0.3} \mathrm{Fe}_{2.35} \mathrm{O}_{4}$ ferrite prepared by modified citrate precursor method. Mater Chem Phys 122: 133

Wang C, Zhang XM, Qian XF, Xie J, Wang WZ, Qian YT (1998) Preparation of nanocrystalline nickel powders through hydrothermal-reduction method. Mater Res Bull 33:1747

Yener DO, Giesche H (2001) Synthesis of pure and manganese-, nickel-, and zinc-doped ferrite particles in water-in-oil microemulsions. J Am Ceram Soc 84:1987

Zhang ZJ, Wang ZL, Chakoumakos BC, Yin JS (1998) Temperature dependence of cation distribution and oxidation state in magnetic $\mathrm{Mn}-\mathrm{Fe}$ ferrite nanocrystals. J Am Chem Soc 120:1800 